Ann. Biol. anim. Bioch. Biophys., r972, 12 (4), 569-579.

\title{
EFFETS D'UN FIL UTÉRIN SUR LES OVIPOSITIONS ET LA COMPOSITION DE L'CEUF CHEZ LA POULE
}

\author{
B. SAUVEUR et L. LACASSAGNE \\ avec la collaboration technique de Huguette Astorne \\ Station de Recherches avicoles, \\ Centre de Recherches de Tours, I. N. R. A., \\ 37380 Nouzilly
}

\section{RÉSUMÉ}

Chez la poule, la présence d'un fil dans la paroi utérine provoque l'expulsion d'œufs sans coquille. Le but de la présente étude a été d'estimer le stade exact d'expulsion, les intervalles entre deux expulsions consécutives ainsi que les modifications éventuelles de composition de l'œuf qui en résultent.

La figure I montre que les ovipositions des œufs sans coquille se situent pour 95 p. Ioo entre I $8 \mathrm{~h}$ et $6 \mathrm{~h}$ ( $80 \mathrm{p}$. Ioo pendant la nuit). Le décalage dans le temps de la classe de plus grande fréquence d'ovipositions semble indiquer que l'œuf sans coquille est expulsé I $3 \mathrm{~h}$ en moyenne avant l'oviposition normale. Les intervalles entre ovipositions d'œufs mous (tabl. 2) ne sont pas statistiquement différents de ceux relatifs aux œufs normaux. Lorsqu'un œuf sans coquille suit un ouf normal, l'intervalle entre les deux ovipositions est de 12 h 31 mn \pm I $6 \mathrm{mn}$.

Outre le poids de l'œuf et celui de la coquille, la teneur en électrolytes de l'albumen est très perturbée (tabl. 3). Les valeurs obtenues sont comparées avec celles déjà enregistrées au cours de la formation normale de l'œuf par Sauveur et MongIN (I97 I). Il apparaît ainsi que les concentrations de sodium et de chlore sont exceptionnellement élevées par rapport au stade d'expulsion; la teneur en potassium de l'albumen est également légèrement accrue.

Ces résultats confirment que la vacuité de l'utérus n'intervient pas dans la régulation des ovulations ; ils montrent par ailleurs que la présence d'un fil utérin modifie les transferts d'électrolytes entre le sang et l'œuf. Ces résultats sont discutés, d'une part en fonction des effets déjà connus des irritants utérins chez les oiseaux, d'autre part en relation avec quelques observations faites chez des mammifères porteurs de dispositifs anticonceptionnels intra-utérins (IUCD).

\section{INTRODUCTION}

Chez ia poule domestique, la présence d'un fil chirurgical dans la paroi de l'utérus provoque l'expulsion d'œufs sans coquille. Ce phénomène, particulièrement intéressant pour l'étude du fonctionnement utérin, a été décrit par SykES (1953, I g62, 
I967) ainsi que par LAKE et GILBERT (I962, I964). Ces auteurs s'accordent à penser que l'absence de coquille est plutôt due à l'expulsion prématurée de l'œuf qu'à un défaut biochimique de précipitation du carbonate de calcium. Par ailleurs, les taux d'ovulation restent inchangés. Malgré l'expulsion prématurée de l'œuf, SykEs (I962, I967) n'observe pas de variation du poids d'albumen mais seulement une diminution de la quantité de calcium déposée sur les membranes coquillères. Nous ne possédons pas de renseignements plus précis sur les modifications éventuelles de composition de l'œuf. C'est pourquoi nous avons étudié la teneur en eau et en électrolytes de l'abumen d'œufs pondus par des poules ayant un fil in utero en essayant par ailleurs d'atteindre une plus grande précision dans l'estimation du moment d'expulsion.

\section{MATÉRIEL ET MÉTHODES}

Nous avons utilisé des poules de type White Leghorn (H. N.) durant leur sixième mois de production; les animaux étaient logés en cages individuelles munies d'enregistreurs automatiques de l'heure de ponte et placées dans un local isolé du point de vue thermique et phonique ; la durée d'éclairement quotidien était de seize heures (de $5 \mathrm{~h}$ à $2 \mathrm{I} \mathrm{h}$ ). Un aliment composé complet pour poules pondeuses a été distribué ad libitum.

La pose d'un fil de lin chirurgical dans la paroi utérine a été effectuée chez six animaux. Pour ce faire, nous avons pratiqué une laparatomie sous anesthésie générale (par courant de $\mathrm{O}_{2}+\mathrm{N}_{2} \mathrm{O}+$ éther) après nous être assurés par palpation à travers le cloaque de la présence dans l'utérus d'un ouf à coquille complète. Cette précaution rend plus facile la pose du fil dans une des parois en assurant la protection de la paroi opposée. L'emplacement choisi a été défini par LAKE et GrLBERT (1964) à mi-distance de l'isthme et du vagin. Les poules témoins-opérées (six) ont subi les mêmes manipulations mais le fil a été seulement passé, puis immédiatement retiré.

Après opération, les animaux ont été replacés dans le bâtiment d'élevage. Tous les œufs mous produits pendant 6 semaines par les poules opérées et ayant pu être récupérés, ont été analysés pour le poids de coquille, la teneur en eau et en électrolytes ( $\mathrm{Na}, \mathrm{K}, \mathrm{Ca}$ et $\mathrm{Cl}$ ) de l'albumen. Ces œufs mous ayant été pondus en quasi-totalité pendant la période nocturne (voir Résultats), leur étude n'a pu être effectuée en moyenne que ro heures après l'oviposition ; il sera tenu compte de ce délai lors de la discussion. Les méthodes de dosages utilisées ont déjà été décrites (SAUVEUR et MongrN, I97r). Les moyennes obtenues ont été comparées à celles enregistrées sur les œufs pondus par les poules témoins-opérées.

\section{RÉSULTATS}

Après six semaines d'expérience, les animaux furent abattus et la position de la boucle vérifiée par autopsie. Chez les six poules, le fil s'est avéré correctement placé; la partie située dans la lumière utérine est apparue calcifiée, ce qui confirme les observations antérieures de LAKE et GILBERT (I964).

\section{Production d'oufs}

Nous avons rapporté au tableau $\mathrm{I}$ les valeurs de l'intensité de ponte et de la fréquence des œufs sans coquille pour les six poules opérées (lot $A)$, les six poules témoins-opérées (lot $B$ ) et un ensemble de trente-cinq poules témoins (lot $\mathrm{C}$ ) non opérées. 
L'intensité de ponte n'apparaît pas modifiée par l'intervention ; le lot opéré A présente des valeurs identiques à celles des animaux témoins (C). Pendant trois semaines au moins, les poules témoins-opérées (lot B) semblent, en revanche, avoir une intensité de ponte supérieure aux deux autres lots ; cependant, le nombre d'animaux est beaucoup trop faible pour qu'une conclusion puisse en être tirée.

TABLEAU I

Caractéristiques de la ponte durant les 6 semaines suivant l'intervention (Egg production during the 6 weeks following operation)

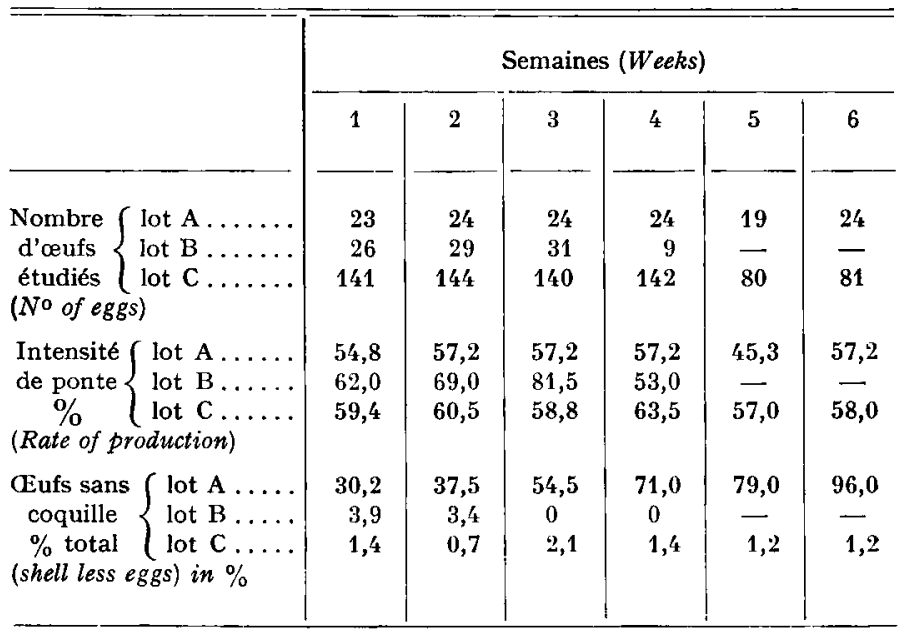

(Groups) lot $\left\{\begin{array}{l}\text { A poules opérées (fil in utero) } \\ \text { B poules témoins-opérées (sham operated controls) } \\ \text { C poules témoins non opérées (intact controls) }\end{array}\right.$

La fréquence des œufs sans coquille augmente régulièrement, au cours des semaines qui suivent l'opération, chez la poule ayant un fil in utero; elle passe ainsi de $30 \mathrm{p}$. Ioo la première semaine à $96 \mathrm{p}$. roo après six semaines. A l'opposé, nous n'observons que des fréquences de I à $3 \mathrm{p}$. roo dans les deux lots $\mathrm{B}$ et $\mathrm{C}$. L'intervention chirurgicale en tant que telle, non suivie de la pose définitive d'un fil, n'exerce donc aucune influence sur la production ultérieure d'œufs sans coquille.

\section{Heures d'oviposition et intervalles entre ovipositions}

La figure I montre pour chaque lot $\mathrm{A}, \mathrm{B}$ et $\mathrm{C}$ la fréquence des ovipositions en fonction de l'heure de la journée. Ces résultats représentent les moyennes de tous les enregistrements effectués durant les 6 semaines d'expérience. Pour les poules avec fil in utero (lot $A$ ) nous avons distingué les ovipositions des œufs sans coquille calcifiée $\left(A_{1}\right)$ de celles des oufs normaux $\left(A_{2}\right)$. 
Il apparaît clairement que la répartition journalière des heures de ponte est la même pour les œufs des poules non opérées (lot $\mathrm{C}$ ), ceux des poules témoins-opérées (lot B) et même pour les œufs normaux pondus par les poules avec fil $\left(A_{2}\right)$; dans les trois cas la fréquence la plus élevée se situe entre 8 et $\mathrm{I} 4 \mathrm{~h}$ et plus précisément entre Io et $\mathrm{I} 2 \mathrm{~h}$. Le temps qui s'écoule entre l'extinction de la lumière à l'origine des ovulations et la classe de plus grande fréquence des heures de ponte est donc de 38 heures en moyenne.
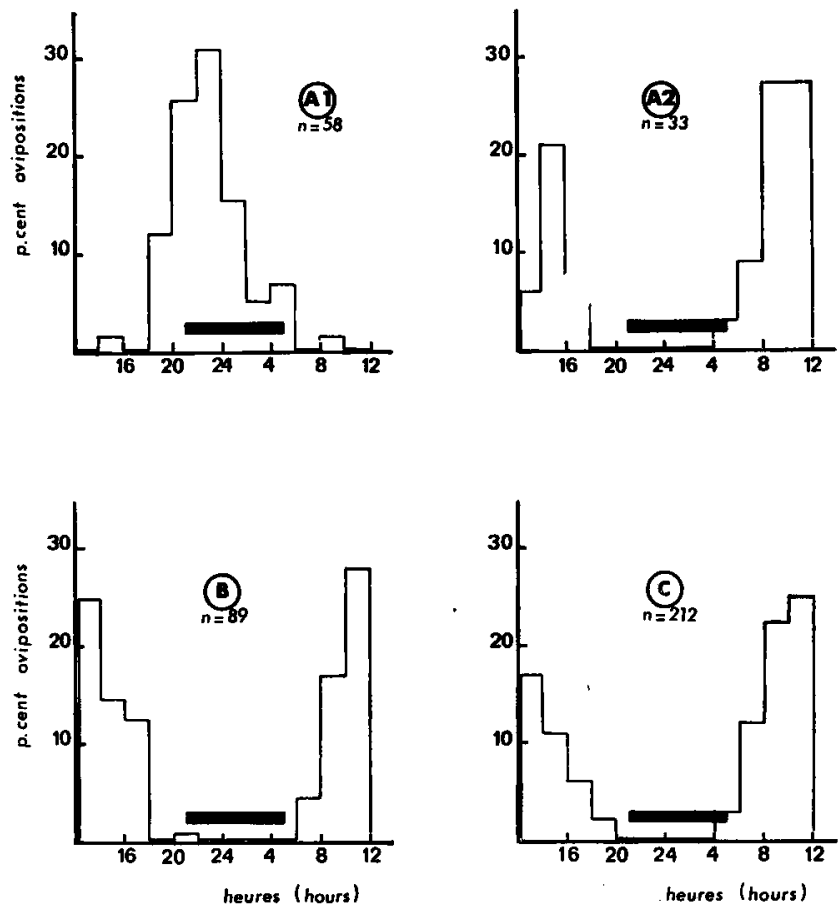

lif. x. - Histogramme de répartition des heures de ponte au cours de la journee (Time repartition of ovipositions through a 24 hours-period)
A : poules avec fil utérin $\left\{\mathbf{A}_{1}\right.$ : œufs sans coquille (shell less eggs)
(hens with uterine thread) $\left\{A_{2}\right.$ : ceufs normaux (normal eggs)
B : poules témoins opérées (sham-operated controls)
C : poules témoins intactes (intact controls)
: période sombre (dark period)
$n$ : nombre d'ovipositions enregistrées ( $N^{\circ}$ of recorded ovipositions)

A l'opposé, les heures de ponte des œufs mous expulsés par les poules avec fil $\left(A_{1}\right)$ se situent entre 20 et $24 \mathrm{~h}$, c'est-à-dire durant la période sombre ; 3 à $4 \mathrm{p}$. Ioo seulement des œufs sans coquille sont pondus durant la période claire, à une heure comparable à celle des cufs normaux. Pour ce groupe $A_{1}$ le décalage moyen $\mathrm{du}$ " pic " de ponte par rapport à l'extinction de la lumière n'est donc plus que de $25 \mathrm{~h}$.

Nous pouvons en déduire que les œufs sans coquille sont expulsés $\mathrm{I} 3 \mathrm{~h}$ environ avant l'oviposition normale.

Nous ävons par ailleurs calculé les intervalles entre ovipositions de deux œufs consécutifs. Les résultats sont ceux du tableau 2 . 
TABLEAU 2

Valeurs moyennes des intervalles entre ovipositions consécutives

(Mean values of time-intervals between 2 consecutive ovipositions)

$$
(\bar{x} \pm \sigma / \sqrt{n})
$$

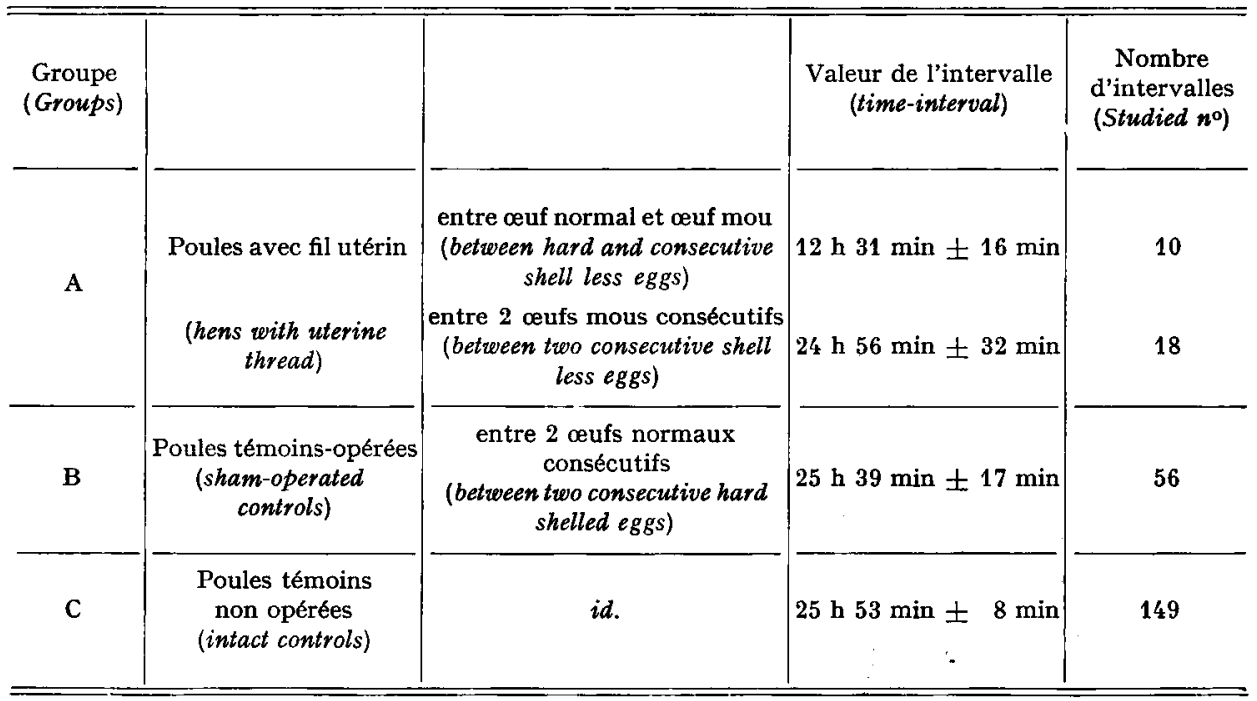

L'intervalle moyen entre ovipositions de 2 œufs sans coquille n'est pas significativement différent de celui observé entre deux œufs normaux ( 24 h 56 contre $25 \mathrm{~h} 39$ en B et 25 h 53 en C). En revanche, lorsqu'un ouf sans coquille est pondu durant la même journée qu'un œuf normal, il est expulsé en moyenne $\mathbf{2} \mathbf{2} \mathbf{h} 30$ min après celui-ci.

Ce résultat peut être rapproché de celui rapporté plus haut : sachant en effet que l'intervalle moyen entre ovipositions est de $25 \mathrm{~h} 53 \mathrm{~min}$ pour les animaux témoins et que les œufs mous sont expulsés I3 heures avant l'oviposition normale, on en déduit que cette expulsion se situe environ à : $26-1_{3} \simeq 1_{3}$ heures après l'oviposition précédente. Ce chiffre s'accorde bien avec les 12 h 30 min ci-dessus mentionnées et montre que l'expulsion d'un œuf sans coquille se produit toujours au même moment (statistiquement) par rapport à l'ovulation, que l'œuf précédent ait été normal ou non.

\section{Effet sur la composition de l'œuf}

L'action d'une expulsion prématurée de l'œuf sur sa composition hydro-minérale est décrite au tábłeau 3. Nous avons fait figurer en bas du tableau les valeurs normales observées durant la formátion de l'œuf chez des animaux de même souche en retenant les stades ro, I2 et I4 $\mathrm{h}$ après oviposition (SAUveur et MONGIN, I97I). Pour tous les paramètres, les vâteurs relatives aux oufs sans coquille sont très différentes de celles des œufs normaux pondus par les poules témoins-opérées. La comparaison statistique des couples de moyennes à l'aide de tests $t$ ne fait que confirmer l'évidence d'une oviposition prémiaturée de l'œuf: 


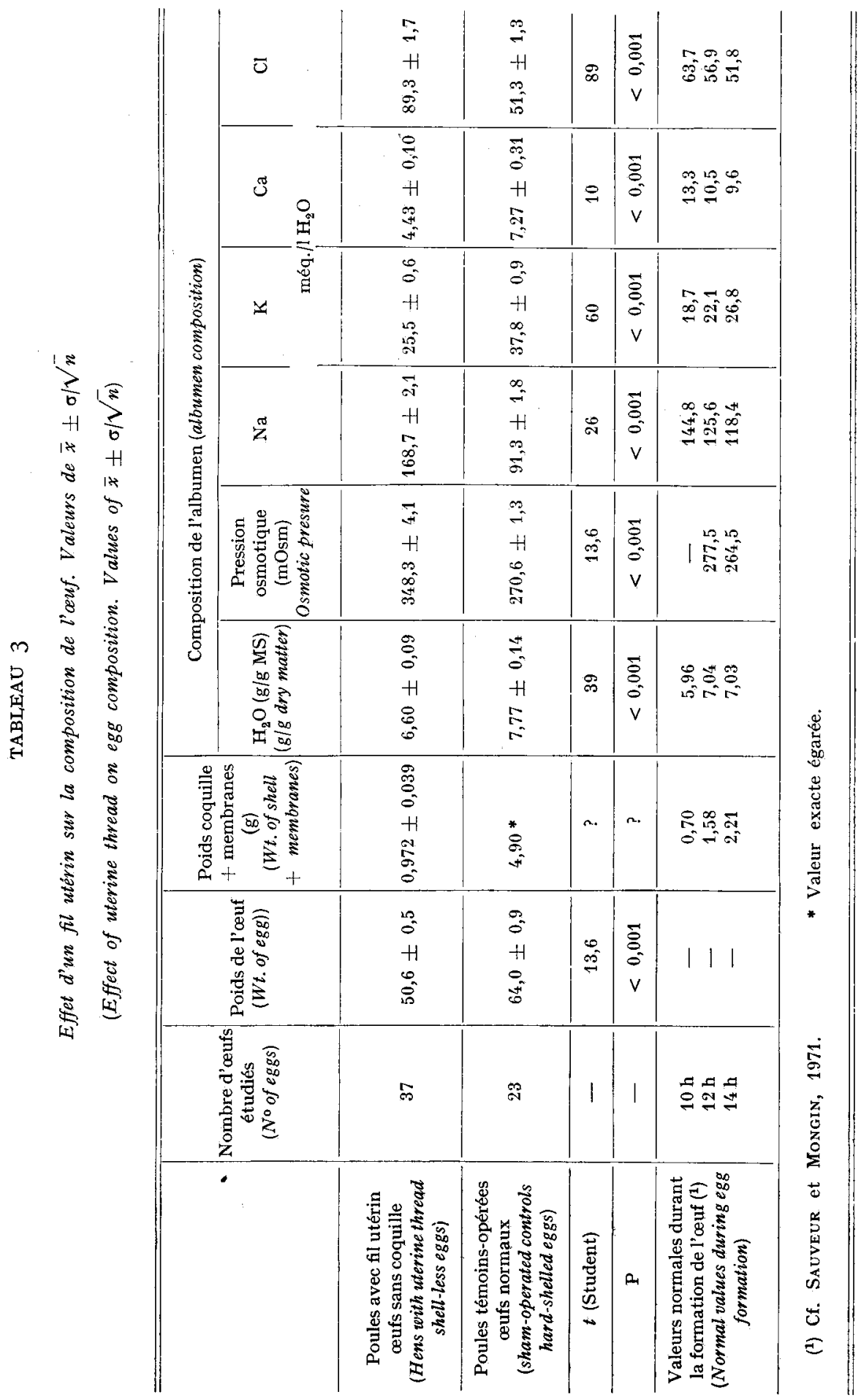


Il est alors intéressant de comparer les valeurs enregistrées sur les œufs pondus sans coquille avec celles observées durant la formation normale de l'œuf. En prenant comme critère le poids de coquille déposé sur les membranes, la formation de l'œuf semble s'être arrêtée entre Io et $\mathrm{I} 2 \mathrm{~h}$ après oviposition de l'œuf précédent. Les valeurs d'hydratation de l'albumen conduisent à la même estimation : elles risquent cependant d'être biaisées puisque l'albumen des oufs mous subissait to $\mathrm{h}$ d'évaporation supplémentaire, à un taux plus élevé du fait de l'absence de coquille.

La teneur en potassium de l'albumen des œufs sans coquille semble indiquer une expulsion légèrement plus tardive ( $\mathrm{I} 2 \mathrm{à} \mathrm{I} 4 \mathrm{~h}$ ). Le résultat le plus surprenant concerne les teneurs de l'albumen en sodium ( 169 méq. $/ 1 \mathrm{H}_{2} \mathrm{O}$ ) et en chlore ( 89 méq. $/ 1 \mathrm{H}_{2} \mathrm{O}$ ) ainsi que la pression osmotique ( 348 mosm). Manifestement, ces trois valeurs ne coïncident absolument pas avec celles que l'on aurait pu attendre entre les stades ro et $\mathrm{I} 4 \mathrm{~h}$ de formation mais leur sont nettement supérieures. En admettant que la ponte se soit produite autour du stade $\mathrm{I} 2 \mathrm{~h}$ (valeur la plus probable, compte tenu des paragraphes précédents) il apparaît un supplément de concentration de 43, I méq./1 pour $\mathrm{Na}$ et 32,4 méq./1 pour $\mathrm{Cl}$; ces valeurs sont en accord avec 1'accroissement de pression osmotique de 78 mosm.

Cet accroissement des teneurs en $\mathrm{Na}$ et $\mathrm{Cl}$ rapportées à l'eau de l'albumen n'est pas un artefact dû à une évaporation éventuelle. En effet, si nous exprimons ces contenus par rapport à la matière sèche de l'albumen nous obtenons :

$$
\begin{aligned}
& \mathrm{Na}=\mathrm{I} \text { 108,3 méq./kg de matière sèche } \\
& \mathrm{Cl}=587,0-1 \\
& \mathrm{~K}={ }_{167,3}-\quad-
\end{aligned}
$$

alors que les valeurs normales obtenues $\mathrm{I} 2 \mathrm{~h}$ après oviposition de $1^{\prime} œ u f$ précédent sont respectivement de $882,7-400, \mathrm{I}$ et 155,8 méq. $/ \mathrm{kg}$ de matière sèche (SAUVEUR et Mongin, I97I). La présence du fil utérin se traduit donc de façon indubitable par un accroissement important du dépôt de sodium et de chlore et, à un bien moindre degré, de potassium dans l'albumen de l'œuf.

\section{DISCUSSION}

L'insertion d'un fil chirurgical dans la paroi utérine de la poule n'affecte pas le taux d'ovulation mais provoque l'expulsion prématurée de l'œuf; sur ces deux points, nos résultats s'accordent avec ceux de Sykfs (I953), Van Tienhoven (I953) et de LAKE, et Gri,BERT (I964).

\section{Heures d'oviposition}

SykEs (I967) affirme que les œufs sans coquille dus à un fil utérin peuvent être pondus à toute heure du nycthémère avec, cependant, une prédominance pour la période nocturne ; LAKE et GILBER'T (I964) montrent au contraire que l'oviposition de tels œufs mous est exclusivement nocturne. Les résultats présents confirment clairement ceux de LAKE, et GILBERT, puisque nous enregistrons environ $80 \mathrm{p}$. IOo des ovipositions d'œufs sans coquille durant la période sombre, avec un " pic " de 
ponte entre 22 et $24 \mathrm{~h}$; de plus, sur les $20 \mathrm{p}$. Ioo d'ovipositions observées pendant la période claire, I5 p. Ioo au moins se produisent entre $\mathrm{I} 8$ et $20 \mathrm{~h}$ ou $5 \mathrm{~h}$ et $6 \mathrm{~h}$, soit durant des périodes très rares chez les animaux témoins.

Par ailleurs, LACASSAGNE (non publié) observe que les œufs naturellement sans coquille sont pondus $24 \mathrm{~h} 38 \mathrm{~min}$ après l'extinction de la lumière ; de même HuGHES et PARKER (I97I) rapportent pour cet intervalle une valeur comprise entre 24 et $26 \mathrm{~h}$. Nous trouvons ici, en présence d'un fil utérin, que la ponte des œufs mous est décalée de $25 \mathrm{~h}$ en moyenne, par rapport à l'extinction de la lumière. Il semble donc que le délai entre la stimulation lumineuse de l'ovulation et l'oviposition de l'œuf mou reste le même, que cette oviposition soit spontanée ou provoquée par un irritant utérin.

\section{Intervalles entre ovipositions}

Il ressort des présentes observations que les intervalles entre les ovipositions consécutives de deux œufs sans coquille ne sont pas statistiquement différents de ceux enregistrés entre deux oufs normaux chez les animaux témoins. Ce résultat n'a pas encore été rapporté à notre connaissance mais il peut être rapproché de 1'observation de WOOD-Gush (I963) suivant laquelle l'expulsion prématurée de l'œuf, due à un fil utérin, n'est accompagnée d'aucun comportement de ponte ou de nidation : celui-ci se produit au moment normalement attendu d'oviposition. Ces deux observations mettent en évidence que la vacuité de l'utérus n'exerce aucune influence sur la régulation du cycle de ponte que ce soit au niveau de l'ovulation (résultats présents) ou du comportement d'oviposition (Wood-GuSH). Dès I935, WARREN et ScotT avaient, d'ailleurs, déjà noté sur ro œufs que l'expulsion prématurée (obtenue par écrasement de l'œuf au début de la formation de la coquille) ne modifie pas l'heure d'ovulation suivante. GILBERT (I967) a rassemblé plusieurs travaux conduisant à une conclusion identique; cet auteur se demande cependant encore si la présence d'un œuf dans l'oviducte participe ou non au contrôle direct de l'ovulation. Il nous semble pourvoir répondre définitivement par la négative en ce qui concerne le rôle éventuel de l'utérus. Ceci n'exclut pas cependant un contrôle indirect par le biais du métabolisme calcique comme le suppose GiLBERT (I969).

En ce qui concerne les intervalles entre 1'oviposition d'un œuf normal et celle d'un cuf sans coquille pondus dans la même journée, la valeur moyenne trouvée ici ( $\mathrm{I} 2 \mathrm{~h}$ 3I $\mathrm{min} \pm \mathrm{I} 6 \mathrm{~min}$ ) est identique à celle enregistrée par LACASSAGNE (non publié) : I $2 \mathrm{~h} 33 \mathrm{~min} \pm 24 \mathrm{~min}$ (calculée sur 26 intervalles séparant un ouf normal d'un œuf mou "spontané "). Cette similitude paraît assez remarquable et conduit à penser que la présence d'un fil utérin recrée les conditions naturelles d'expulsion des œufs sans coquille.

\section{Composition de l'ceuf}

SYKES (I962 et I967) compare les poids de coquille des œufs mous à ceux normalement rencontrés au cours de la formation de 1'œuf (BURMESTER et al., I939) et estime ainsi que l'œuf a dû être expulsé environ Io h (de 5 à $\mathrm{I} 5 \mathrm{~h}$ ) après l'oviposition précédente. Les résultats présents permettent une estimation plus précise puisque nous trouvons : 
I3 h d'après les heures d'oviposition,

I2 $\mathrm{h} 30$ min d'après les intervalles entre ovipositions,

de II $\mathrm{h}$ à $\mathrm{I} 2 \mathrm{~h}$ d'après le poids de coquille et l'hydratation de 1'albumen comparés aux valeurs enregistrées par SAUVEUR et MONGIN (I97I) sur des animaux de même souche.

Compte tenu de la remarque déjà faite sur la sous-estimation probable de la teneur en eau de l'albumen, le stade $\mathrm{I} 2 \mathrm{~h}-\mathrm{I} 3 \mathrm{~h}$ semble être l'estimation la plus probable du moment d'expulsion. Sykes (1967) estime que la phase de "plumping " doit être terminée au moment de l'expulsion ; ceci semble en effet être le cas puisqu'à I2 $\mathrm{h}$ la phase rapide d'hydratation de l'albumen est achevée. Il est cependant faux de parler d'accumulation maximum d'albumen lors de l'expulsion, sachant qu'une phase lente d'hydratation se serait encore poursuivie durant les douze dernières heures de formation de l'œuf (SAUveur et Mongin, I97I).

LAKE et GILBERT (I964) ne disent rien de l'effet du fil utérin sur le poids de l'œuf ; Sykes (I967) aboutit au résultat assez surprenant que le poids de 1'œuf n'est pas modifié, la réduction du poids de coquille semblant être approximativement compensée par une élévation du poids du jaune. Nos résultats sont en opposition avec ceux-ci puisque nous enregistrons une baisse de $\mathrm{I}_{4} \mathrm{~g}$ du poids de l'œuf dont $4 \mathrm{~g}$ au moins sont attribuables à la coquille. L'évaporation survenue entre la ponte et la pesée de l'œuf n'est certainement pas responsable de cette réduction de poids de ro $\mathrm{g}$ et il est plus vraisemblable d'admettre que la présence du fil utérin entraîne une réduction d'hydratation de l'albumen. Nous n'avons pas enregistré ici le poids du jaune.

La partie la plus surprenante des présents résultats concerne les concentrations exceptionnellement élevées de $\mathrm{Na}$ et $\mathrm{Cl}$ dans l'albumen des œufs expulsés prématurément. A un moindre degré, la teneur en $\mathrm{K}$ est également augmentée. Ce résultat particulier nous semble original et nous n'avons pu trouver aucune référence traitant de l'action d'un fil chirurgical sur la composition électrolytique des sécrétions utérines chez les oiseaux.

De telles références existent pour les mammifères : KAR et al. (I964) observent chez la ratte ovariectomisée et traitée à l'œstradiol une augmentation des teneurs en $\mathrm{Na}$ et $\mathrm{K}$ du liquide utérin sous l'effet d'un dispositif anticonceptionnel intra-utérin (IUD) ; cependant, chez des rattes à cycles normaux, BATTA et al. (I969a) ne retrouvent pas d'effet similaire. De même, KAR et al. (Ig68) n'enregistrent aucune variation significative de teneur en $\mathrm{Na}$ ou $\mathrm{K}$ du liquide intra-utérin chez les femmes portetuses d'IUD. Dans tous les cas les variations individuelles sont très importantes. Plus intéressants pour nous sont les travaux de DANIEL et DANIEI, (I959) et de DANIEI, (I964) relatifs au contrôle des transferts d'ions in utero d'une part par la progestérone, d'autre part par les contractions des muscles lisses. Ces auteurs montrent en effet que les contractions utérines s'accompagnent, chez la lapine, d'une perte tissulaire de potassium et que le gain tissulaire de sodium peut simultanément être masqué par une perte de liquide interstitiel.

LAKE et GILBERT (1964) admettent que la présence d'un fil utérin chez la poule modifie la tonicité musculaire, sans pouvoir dire s'il s'agit d'un relâchement ou, au contraire, d'une hyperactivité. BATTA et al. (I969 b) observent que, chez la ratte, le liquide intraluminal provenant d'une corne utérine porteuse d'un IUD est susceptible de provoquer la contraction d'un muscle lisse à un degré beaucoup plus 
important que le liquide de la corne opposée sans IUD. Si la réponse à la présence d'un "IUD " est la même chez les oiseaux, il devient possible d'expliquer les variations de composition électrolytiques de l'albumen par la simple intervention des phénomènes de contraction. L'accroissement des concentrations de $\mathrm{Na}$ et $\mathrm{Cl}$ dans l'albumen serait alors le résultat d'une diminution de volume extracellulaire, tandis que la légère augmentation de $\mathrm{K}$ correspondrait à une perte tissulaire de cet élément.

Une telle hypothèse demande évidemment vérification. Cependant, il ne nous semble pas impossible a priori de rapprocher les problèmes de transferts minéraux utérins des oiseaux de ceux des mammifères puisque, outre des teneurs voisines en ions $\mathrm{Na}$ et $\mathrm{K}$ des sécrétions utérines, on a même décrit la précipitation de carbonate de calcium sur des dispositifs anticonceptionnels utérins chez l'Homme (Yang et YANG, I97I ; HowARD, I97I ; TÖTtERMAN et ARSTILA, I97I).

Reçu pour publication en juin 1972.

\section{SUMMARY}

\section{FEFECTS OF A UTERINE THREAD ON OVIPOSITION AND EGG COMPOSITION IN THE HEN}

The presence of a thread in the uterine wall of the hen causes the expulsion of eggs without a shell. The object of this study was to estimate the exact stage of expulsion and the interval between two to consecutive expulsions, as well as changes in egg composition which may result.

Figure I shows that the oviposition of eggs without a shell occurs between I $8 \mathrm{~h}$ and $6 \mathrm{~h}$ in $90 \mathrm{p}$. I 100 of cases ( $80 \mathrm{p}$. Ioo during the night). The time difference of the class having the highest frequency of ovipositions seems to indicate that the egg without a shell is expulsed on an average of $\mathrm{I}_{3} \mathrm{~h}$ before normal oviposition. The intervals between soft egg ovipositions (table 2) are not statistically different from those for normal eggs. When an egg without a shell follows a normal egg, the interval between the two ovipositions is $12 \mathrm{~h} 3 \mathrm{I} \mathrm{mn} \pm \mathrm{I} 6 \mathrm{mn}$.

Besides the weight of the egg and the shell, the albumen electrolyte content is disturbed by premature expulsion (table 3). The values obtained are compared with those already recorded during normal egg formation (SAUveur and MoNGIN, I97I). Sodium and chloride concentration seems to be exceptionally high in relation to the expulsion stage; albumen potassium content also increases slightly.

These results confirm that the empty uterus does not play a role in ovulation regulation, and that the presence of a uterine thread changes electrolyte exchange between the blood and the egg. The results are discussed in relation to the already-known effects of uterine irritants on birds, and in relation to some observations on mammals having intra-uterine anti-conceptive devices (I.U.C.D).

\section{RÉFÉRENCES BIBLIOGRAPHIQUES}

Batta S. K., Walia H. B., Chaudury R.-R., I969a. The intraluminal fluid volume and electrolyte concentration in rat uteri with an intra uterine silk thread suture in one horm. Ind. Jour. Med. Res., 57, I706-г710.

Batta S. K., Chaudury R. R., I969 b. Observation on the intraluminal fluid obtained from rat uteri with an intrauterine device in one horm. Ind. Jour. Med. Res., 57, I7II-I723.

Burmester B. R., Scott H. M., Card L. E., 1939. Proc. VIIth World's Poulty Sci. Congr., 99-1o3. DANiEl E.-E., The interconnection between active transport and contracture in uterine tissues. Can. J. Physiol. Pharmacol., 42, 453-495. 
Daniel E. E., Daniel Betty N., 1959. Effects of contractile stimuli on exchanges of electrolytes between uterine tissues and a saline-bicarbonate medium. Can. J. Biochem. Physiol., 37, I27-I48.

GrLbert A. B., I967. Formation of the egg in the domestic chicken. In Mc LAREN A., Advances in reproductive Physiol., 2, Logos Press, London, III-180.

Gizbert A.B., I969. The effect of a foreign object in the shell gland on egg production of hens on a calcium-deficient diet. Br. Poult. Sci., 10, 83-88.

Howard Geraldine, I97x. The significance of calcium deposits occuring on intrauterine devices. $J$. Obst. Gynaecol. Brit. Com., 78, 86r-862.

Hughes B. L., Parker T. E., I97I. Time of oviposition of shell-less eggs. Poult. Sci., 50, 1509-15II

Huston T. M., Nalbandov A. V., I953. Neurohumoral control of the pituitary in the fowl. Endocrinology., 52, I49-I56.

Kar A. B., Goswani A., Kamboj V. P., Chowdury S. R., I964. Effect of foreign body on the response of the uterus of ovariectomized rats to oestrogen. Steroids, 4, I59.

Kar A. B., Engineer A. D., Gorl R., Kamboj V. P., Dasgupta P. R., Chowdury S. R., I968. Effect of intrauterine contraceptive device on biochemical composition of uterine fluid. Am. J. Obst. Gynaecol., 101, 966-970.

LAKE P. E., Gilbert A. B., I962. Factors affecting uterine motility in the domestic hen. J. Reprod. Fert., 4, $2 \mathrm{Ir}$.

Lake P. E., Gilbert A. B., r964. The effect on egg production of a foreign object in the lower oviduct regions of the domestic hen. Res. Vet. Sci., 5, 39-45.

Sauveur B., Mongin P., r97I. Étude comparative du fluide utérin et de l'albumen de l'œuf in utero chez la poule. Ann. Biol. anim. Bioch. Biophys., 11, 213-224.

Sykes A. H., I953. Premature oviposition in the hen. Nature, 172, rog8-rog9.

Sykes A. H., 1962. Effect of a uterine irritant on egg formation in the fowl. J. Reprod. Fert., 4, 2 I4.

Sykes A. H., I967. The effect of a uterine irritant on the composition of the egg in the fowl. J. Endocrin., 37, I03-I04.

VAN Thienhoven A., 1953. Anat. Rec., 115, 374-375.

Tötterman L. E., Arstila A. A., 1971. Deposit on the surface of IUDs. Ann. Chirur. Gynaecol. Fen., 60, II5-II8.

Warren D. C., Scote H. M., 1935. The time factor in egg formation. Poult. Sci., 14, 195-207.

Wood-Gush D. G. M., I963. The control of the nesting behaviour of the domestic hen. I. The role of the oviduct. Anim. Behav., 11, 293-299.

YANG T. S., YANG W. H., I97I. Calcium carbonate deposition on intrauterine contraceptive devices. Am. J. Obst. Gynaecol., 109, 664-666. 